\title{
Pancreatic Tumour Mimicking Pregnancy; a Para-Neoplastic Syndrome: A Case Report and Review of Relevant Literatures
}

\author{
Omotoso A $\mathrm{J}^{1}$, Nnoli $\mathrm{Ma}^{1}$, Bassey I $\mathrm{E}^{1}$, Akintomide $\mathrm{A} \mathrm{O}^{1,2}$, Odusolu $\mathrm{P}^{2,3}$, Udor \\ $\mathrm{A}^{2,3}$, Agan $\mathrm{Tu}^{2,3}$, Ngim $\mathrm{O} \mathrm{E}^{3}$ \\ ${ }^{1,2}$ Department of Pathology, University of Calabar Teaching Hospital Calabar \\ ${ }^{3}$ Department of Obstetrics and Gynaecology, University of Calabar Teaching Hospital, Calabar \\ ${ }^{4}$ Department of Surgery, University of Calabar Teaching Hospital Calabar
}

\begin{abstract}
A young lady presented with epigastric pain and amenorrhea of two months duration. Pregnancy test was positive, however, ultrasonography showed an empty uterus. Abdominal ultrasound revealed nodules on the liver with possible lesions in the pancrease. A CT scan subsequently revealed a pancreatic tumour with metastasis to different organs. Patient later developed jaundice and died before any intervention could be made. Pancreatic tumour is often diagnosed late due to their non specific symptoms. After the diagnosis is made the mortality is high irrespective of the pathologic and clinical stages. Paraneoplastic syndrome is often seen with oat cell carcinoma of the lung with significant production of cortisol. The presence of high level of HCG in woman is often associated with pregnancy or other trophoblastic diseases. It could also be seen in patients with pancreatic cancer.
\end{abstract}

Key Words: Paraneoplastic, Pancrease, CT SCAN, HCG

\section{Background}

Pancreatic cancer is the fourth most common cause of cancer death in men and women in the US. Pancreatic cancer is the fourth most common cause of cancer-related deaths in the United States ${ }^{1}$ and the eighth worldwide. ${ }^{1}$ Pancreatic cancer has an extremely poor prognosis: for all stages combined, the 1- and 5-year relative survival rates are $25 \%$ and $6 \%$, respectively; ${ }^{1}$ for local disease the 5 -year survival is approximately $15 \%$, while the median survival for locally advanced and for metastatic disease, which collectively represent over $80 \%$ of individuals, is about 10 and 6 months respectively. ${ }^{2}$ Individuals vary, however - some are only diagnosed when they are terminally ill already and therefore only have a few days or weeks. Others have slower progression and may live a couple of years even if surgery is not possible. Men are $30 \%$ more likely to get pancreatic cancer than are women. Persons of African nationality or descent are more likely to develop pancreatic cancer than white persons and it is commoner among black Americans than white Americans ${ }^{10}$

Early pancreatic cancer often does not cause symptoms and the later symptoms are usually nonspecific and varied. Therefore, pancreatic cancer is often not diagnosed until it is advanced. ${ }^{3}$ Common symptoms include: Pain in the upper abdomen that typically radiates to the back (seen in carcinoma of the body or tail of the pancreas), heartburn - acid stomach, poor appetite or nausea and vomiting, diarrhea, loose stools, significant weight loss (cachexia), painless jaundice (yellow tint to whites of eyes (sclera) or yellowish skin, possibly in combination with darkened urine) when a cancer of the head of the pancreas $(75 \% \text { of cases })^{4}$ obstructs the common bile duct as it runs through the pancreas. This may also cause pale-colored stool and steatorrhea. The jaundice may be associated with itching as the salt from excess bile can cause skin irritation.

Trousseau sign is also another symptom seen in patient with pancreatic cancer. In this condition blood clots are formed spontaneously in the portal blood vessels, the deep veins of the extremities, or the superficial veins in any location in the body. Diabetes mellitus or elevated blood sugar levels may also be seen. Many patients with pancreatic cancer develop diabetes months to even years before they are diagnosed with pancreatic cancer, suggesting new onset diabetes in an elderly individual may be an early warning sign of pancreatic cancer. $^{4}$

Symptoms of pancreatic cancer metastasis could present in different ways depending on the organs affected. Typically, pancreatic cancer first metastasizes to regional lymph nodes, and later to the liver or to the peritoneal cavity and later to the lungs; it rarely metastasizes to bone or brain ${ }^{5}$.

Paraneoplastic Syndromes are symptom complexes in cancer-bearing individuals that cannot readily be explained, either by the local or distant spread of the tumor or by the elaboration of hormones indigenous to the tissue from which the tumor arose. These occur in about $10 \%$ of persons with malignant disease ${ }^{6}$. Despite their relative infrequency, paraneoplastic syndromes are important to recognize, for several reasons: They may represent the earliest manifestation of an occult neoplasm. The affected patients may present with significant problems which may even be lethal. They may mimic metastatic disease and therefore confound treatment. 
Recent medical advances have improved the understanding, diagnosis, and treatment of paraneoplastic syndromes. These disorders arise from tumor secretion of hormones, peptides, or cytokines or from immune cross-reactivity between malignant and normal tissues. Paraneoplastic syndromes may affect diverse organ systems, most notably the endocrine, neurologic, dermatologic, rheumatologic, and hematologic systems. The most commonly associated malignancies include small cell lung cancer, breast cancer, gynecologic tumors, and hematologic malignancies. In some instances, the timely diagnosis of these conditions may lead to detection of an otherwise clinically occult tumor at an early and highly treatable stage. Because paraneoplastic syndromes often cause considerable morbidity, effective treatment can improve patient quality of life, enhance the delivery of cancer therapy, and prolong survival. Treatments include addressing the underlying malignancy, immunosuppression (for neurologic, dermatologic, and rheumatologic paraneoplastic syndromes), and correction of electrolyte and hormonal derangements (for endocrine paraneoplastic syndromes).

Human chorionic gonadotropin (HCG) is formed from two different protein subunits encoded by separate genes. The first is the $\alpha$ subunit that is shared by all members of the pituitary class of glycoprotein hormones including HCG, luteinizing hormone (LH), follicle-stimulating hormone (FSH), and thyroidstimulating hormone (TSH). The second, the $\beta$ subunit, is unique for each of these hormones. Production of HCG is found in trophoblastic tumors (choriocarcinomas, testicular embryonal carcinomas, and seminomas) and, uncommonly, in tumors of the lung and pancreas. Clinical syndromes caused by excessive HCG production are age-dependent. In younger children, precocious puberty, caused by HCG stimulation of ovarian function, is seen. In adult males gynecomastia is a common occurrence. Hyperthyroidism may develop from an interaction of HCG with the thyroid-stimulating hormone receptor (TSHR) particularly when $\beta$-HCG is expressed at high levels. $\beta$-HCG does not activate the TSHR under normal physiologic circumstances, but when expressed at high levels, the TSHR can be continuously activated leading to hyperthyroidism. ${ }^{4}$

Removal or effective therapy for the underlying tumor is the most effective therapy for clinical syndromes caused by excessive $\beta$-HCG production. Hyperthyroidism can be treated short-term with thionamide therapy if there is belief that chemotherapy or other strategies to treat the underlying malignancy are likely to be effective. In patients with less responsive tumors, surgery or radioactive iodine may be required.

\section{Case Report}

A 32 year -old para $2+1$ female patient presented at the medical out patient's clinic with the history of fullness of abdomen with epigastric pain. After examination, an assessment of gastritis was made and was treated for such. She however, presented two weeks later with same presentation and amenorrhea, easy fatigability, easy fullness and dyspnea on exertion. Pregnancy Test using early morning urine and blood samples were both positive. Pelvic ultrasound scans both transvaginal and suprapubic showed empty uterus. An abdominal ultra sound however revealed nodular lesions on the liver with primary tumour likely from the pancrease. A CT scan showed a pancreatic tumour with extensive metastasis to the liver and the lungs. The patient later developed jaundice which gradually got worsened. The patient however died 7 days after the CT scan report before any surgical intervention.

Autopsy findings revealed a young, deeply icteric patient. She was not pale, not cyanosed. Peripheral lymph node was not seen but with bilateral pitting oedema to the thigh and extension to the trunk. The serous cavities were filled with excess serous fluid. The organs were in their anatomic passion with omental caking.

There was massively enlarged pancrease with greyish white nodular tumour affecting the entire mass. The cut surface showed areas of haemorrahgic and necrosis especially at head of the pancrease. The paraaortic lymph nodes were matted together. There were tumour metastases to the liver. These lesions were geographically distributed all over the liver, measuring $0.5-2.5 \mathrm{~cm}$. with central area of necrosis (umbilication). Tumour deposits were also seen on the kidney and the lungs. The histology of the lesions was similar showing malignant proliferation of epithelial cell forming glands- adenocarcinoma. These cells were pleomorphic, hyperchromatic with coarse chromatin pattern. There was tumour necrosis and haemorrhage at the background. 


\section{Scan Result}

Figure I. Axial CT of the pelvis at the level of the uterus

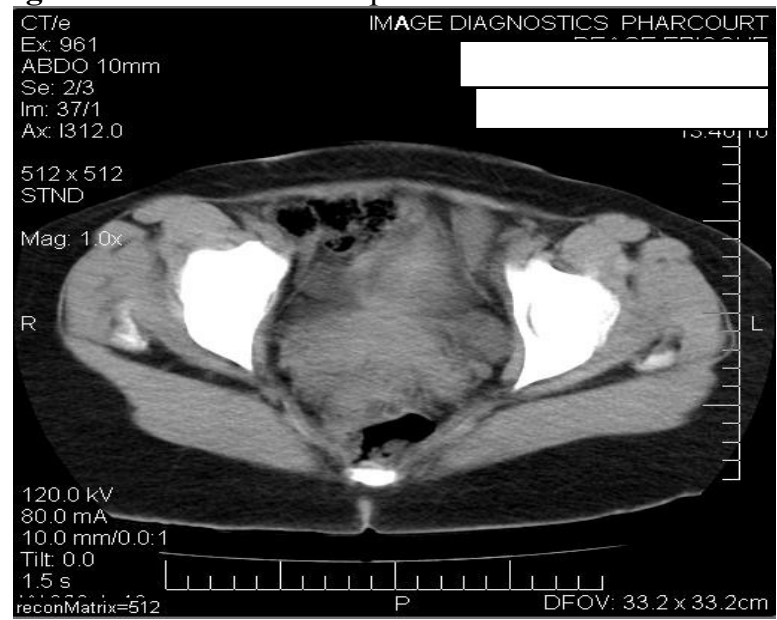

Figure II. Contrast enhanced Axial CT of the upper abdomen at the level of the pancreas.

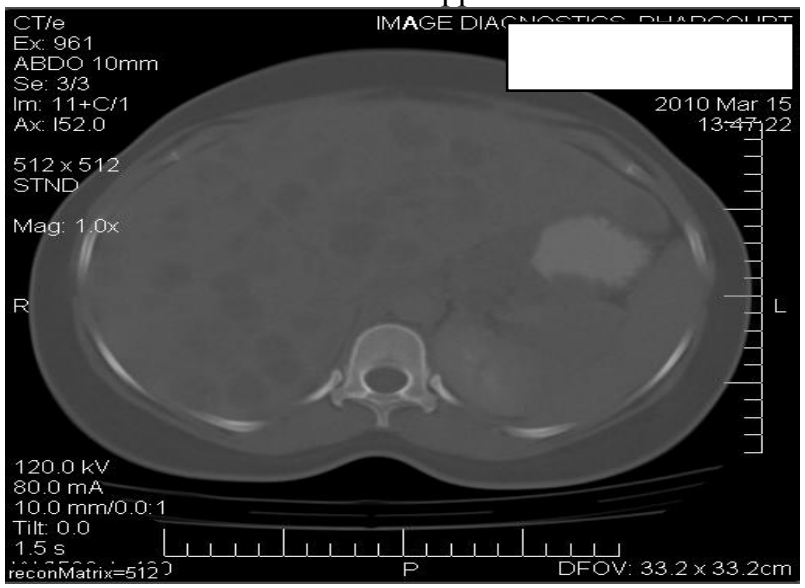

Figure III: Non contrast enhanced Axial CT of the upper abdomen at the level of the pancreas.

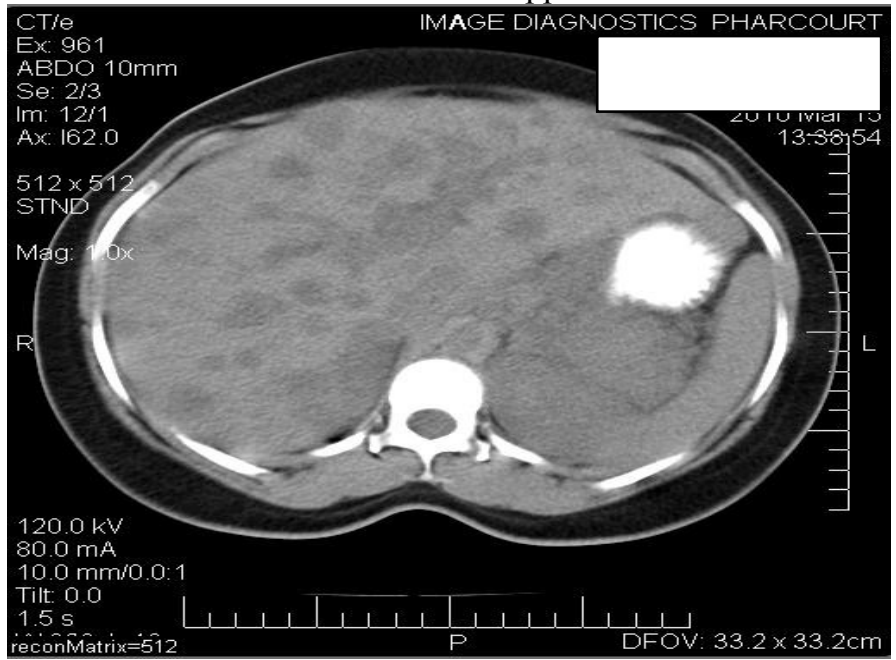




\section{Autopsy Photograph}

Figure IV: section showing nodular enlarged pancrease with necrosis at the head of the pancrease with dilated blood vessels and matted lymph.

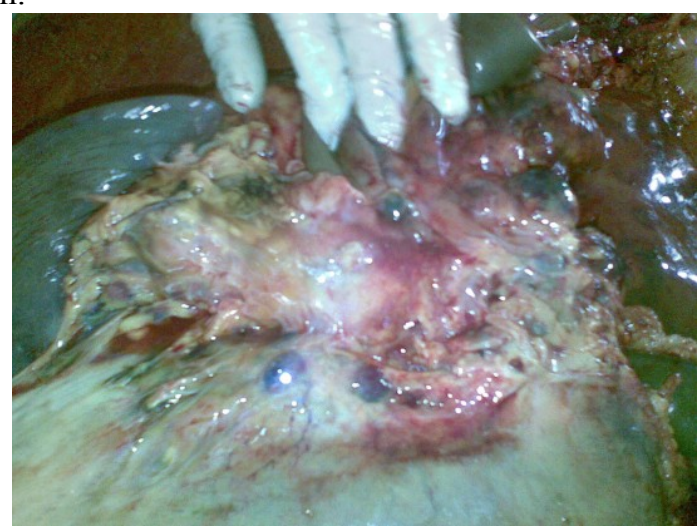

Figure V: Section showing diffuse metastatic tumour to the liver with haemorrhage and necrosis

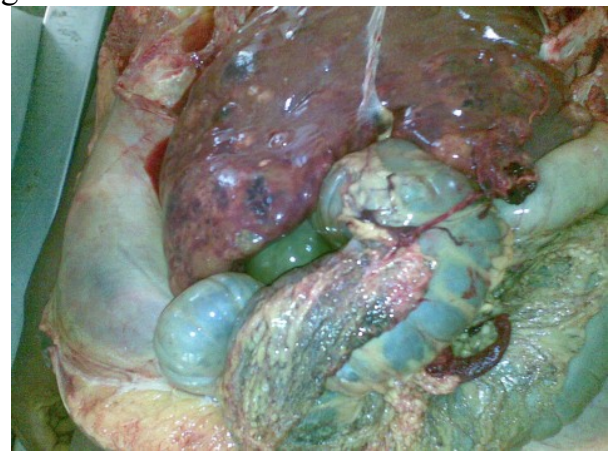

Figure VI: Umbilication seen on the surface of tumour deposit to the liver showing metastasis of the pancreatic tumour to the liver.

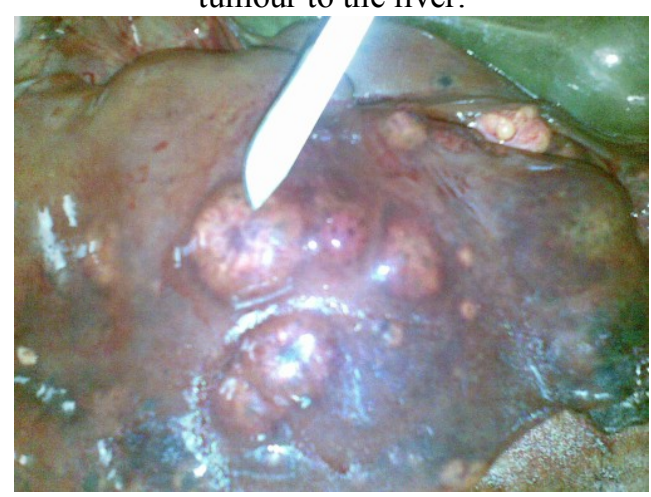

Figure VII: Section showing tumour deposit on the cut surface of the lung

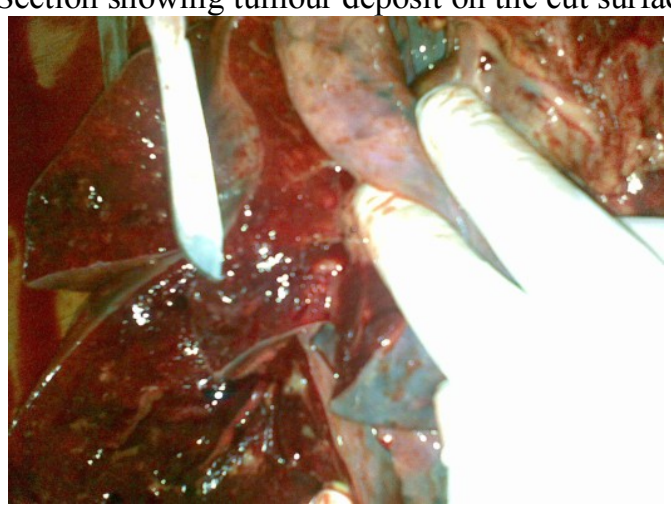




\section{Discussion}

The occurrence of pancreatic carcinoma in a young patient is rare however there are few reported cases. Marinoni et al reported a case of pancreatic adenocarcinoma, with lung and liver metastases, diagnosed in a woman in her third trimester of pregnancy ( 28 weeks). Ultrasound and magnetic resonance imaging scans were carried out and pancreatic mass biopsy during endoscopic retrograde cholangiopancreatography was performed. Severe preeclampsia and fetal growth restriction occurred. A female infant was delivered by cesarean section at 30 weeks of gestation for worsening of maternal clinical conditions and hepatic and pancreatic tests. The patient died 50 days after delivery ${ }^{7}$. Although pancreatic cancer is a rare condition, it should be suspected when an epigastric pain and laboratory parameter is suggestive of biliary obstruction because the presentation is similar with the index case.

Delayed gastric emptying has been observed in some patients with histologically proved pancreatic cancer without evidence of obstruction. Cara et al described a case of a 73-year-old woman who had signs and symptoms of delayed gastric emptying. Workup included a gastric emptying study that showed $95 \%$ retention of the radiolabeled test meal after 2 hours (normal result, $<70 \%$ ). Abdominal computed tomography showed findings consistent with a pancreatic mass. Indirect immunofluorescence study of the patient's serum showed staining of Purkinje cell nuclei and prompted further evaluation for malignant masses. No other cause for the patient's gastroparesis was found, including obstruction. This case illustrates how gastroparesis with no other apparent cause, particularly in elderly patients, may represent a paraneoplastic syndrome. Pancreatic cancer should be included in the list of occult carcinomas that can manifest in this manner ${ }^{8}$.

Alatise et al had an extensive study of 96 patients diagnosed of pancreatic cancer in Ife, Nigeria. Pancreatic cancer constitute $2.1 \%$ of cancer seen in the region with median age of 55 and male :female ratio of $2: 1$. More than $96 \%$ of the cases involved the entire pancrease which is similar to the index case ${ }^{3}$. He rather concluded in other studies that pancreatic cancer will remain a challenging problem into the $21^{\text {st }}$ century worldwide and that early detection, sceening and staging of patients will be expected to facilitate progress in the management of patients with this disease ${ }^{9}$.

\section{Conclusion}

It is important to note that a very high index of suspicion is expected in any patient presented with vague abdominal pain. Clinician should endeavour to look outside the box in an amennorhic patient with positive pregnancy test.

\section{References}

[1]. Hruban RH, Offerhaus GJA, Kern SE. Familial pancreatic cancer. In Cameron JL, editor, Pancreatic Cancer Hamilton, London, Ontario, BC Decker, Inc., 2001; 25-36

[2]. Jemal A, Murray T, Samuels A, Ghafoor A, Ward E, Thun MJ. Cancer statistics 2003. A Cancer Journal for Clinicians. 2003 ; 53 : $5-26$

[3]. OI Alatise, OO Lawal, OT Ojo Challenges of Pancreatic Cancer Management in a Resource Scarce Setting East and Central African Journal of Surgery, Vol. 15, No. 2, July-December, 2010, pp. 52-58

[4]. Lorraine C. Pelosof, David E. Gerber. Paraneoplastic Syndromes: An Approach to Diagnosis and Treatment. Mayo Clin Proc. 2010 September; 85(9): 838-854.

[5]. Darnell R, Posner J: Paraneoplastic syndromes involving the nervous system. N Engl J Med 2003; $349: 1543$.

[6]. Robbins and Cotran pathologic basis of disease. Vinay Kumar... [et al.]; Saunders, an imprint of Elsevier Inc. 8th ed., 2010

[7]. Marinoni E, Di Netta T, Caramanico L, Tomei B, Moscarini M, Di Iorio R. Metastatic pancreatic cancer in late pregnancy: a case report and review of the literature. J Matern Fetal Neonatal Med. 2006 Apr;19(4):247-9

[8]. Caras S, Laurie S, Cronk W, Tompkins W, Brashear R, McCallum RW Am J Med Sci. 1996 Jul;312(1):34-6.Case report: pancreatic cancer presenting with paraneoplastic gastroparesis

[9]. Alatise OI, Ndububa DA, Ojo OS, Agbakwuru EA, Arowolo AO. Pancreatic cancer in Nigeria: Past, Present and future: Nig Journal of Gastro and Hepatology 2009; 1 (2); 61-74

[10]. Silverman DT, Hoover RN, Brown LM, Swanson GM, Schiffman M, Greenberg RS, Hayes RB, Lillemoe KD, Schoenberg JB, Schwartz AG, Liff J, Pottern LM, Fraumeni JF Jr. Why do Black Americans have a higher risk of pancreatic cancer than White Americans? Epidemiology. 2003 Jan;14(1):45-54. 